\title{
COVID-19 Q\&A: Keeping a cancer core going
}

\author{
Jennifer Koblinski explains how the Cancer Mouse Models Core at Virginia Commonwealth University (VCU) \\ Massey Cancer Center (MCC) has kept its facility up and running in the face of the ongoing coronavirus pandemic.
}

\begin{abstract}
What is your role at your facility, what animals do you care for, and what are they used for?

I am the director of the Cancer Mouse Models Core (CMMC) at Virginia Commonwealth University (VCU) Massey Cancer Center (MCC). The CMMC facilitates in vivo preclinical/translational research by developing patient-derived xenografts (PDX), providing assistance with mouse models of cancer and in vivo drug testing, as well as providing assistance with related pathology/histology services including multiplex immunohistochemistry and quantitative digital pathology for VCU and MCC investigators.
\end{abstract}

\section{How has your facility responded to COVID-19?}

Early in March, the VCU Animal Care and Use Program along with the Division of Animal Resources (DAR) held a Zoom meeting to discuss the best plan forward to address the suspected challenges we would face. They asked for volunteers to learn husbandry in case there would be a shortage of caretakers. Many people, including my staff, volunteered.

We were asked to cryopreserve any important mouse colonies and stop breeding animals to keep everything at minimal mouse maintenance. Imports and exports of mice were stopped effective 3/19/2020. My core reached out to all our users to inform them that we would finish all on-going projects, but no new projects would be started unless they were deemed essential or COVID-19 related. This has been respected by our investigators.

We currently stopped taking any patient's samples to develop PDX models. All our PDX models have been frozen down. We have two technicians taking turns coming in daily to the animal facility to finish the studies that had begun before March 13 and

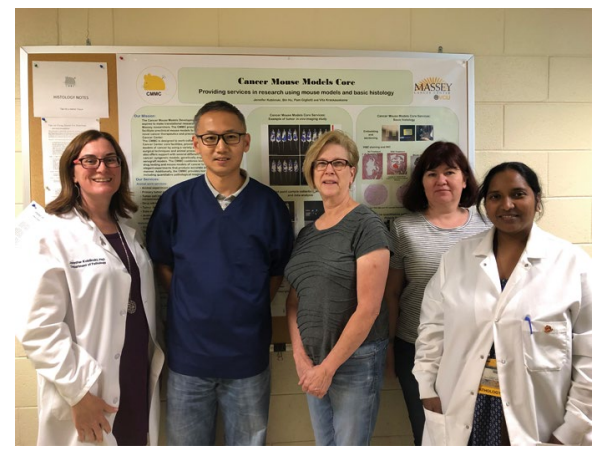

The VCU MMC Cancer Mouse Models

Core. From left to right: Jennifer Koblinski

(Director), Bin Hu (Manager), Pam Gigliotti

(Technician), Vita Kraskauskiene (Technician),

Madhavi Puchalapalli (Technician)

[Not pictured: Chau Gurung (Technician)].

to continue adherence to animal protocols. This helps us keep social distancing in place by only having one person in at a time. If they know another person is in the animal room, they will take the mice to a procedure room if necessary, to avoid any other personnel.

We are communicating daily via group emails about what work has been done that day, how the animals are doing, and what needs to be done the next day in case someone gets sick. We are also holding group meetings 2 times a week by Zoom. Importantly, we get updates daily from the DAR indicating the husbandry level in which they are operating, which thankfully is still at green, meaning there is no change from normal husbandry procedure. The levels of operation are posted and available to all animal users.

\section{Did you feel prepared?}

The guidance from VCU Office of the Vice President for Research and Innovation,
MCC leadership, and the DAR have made our core feel very prepared. The biggest challenge is communicating to others about what is deemed essential research since everyone feels their research is essential. However, I feel VCU has done best in making that as clear as possible. I believe if something like this were to happen again then it would be more seamless, since we all understand better the actions that need to be taken.

\section{How will things return to normal when the time comes?}

As soon as we can resume normal research practices, we will be able to start any new studies. Hopefully, there will not be a shortage of mice available from vendors. If there is no shortage, then it will not take us long to get back up and running with our normal operations for many studies. However, to regenerate our NSG mouse colony, it will take 3-6 months to recover to the level we were at previously. Additionally, the studies relying on PDX samples will take several months to get enough mice to do the experiment. The impact on the users of our transgenic core will be greater. It could take them 3-12 months to regenerate the mice needed to carry out their research projects.

\section{Anything else you'd like to share?} This is stressful to all investigators, especially in these times when the National Cancer Institute funding is so competitive. Additionally, it is stressful to junior faculty with tenure clocks ticking. However, we are all coming together to help each other in these times.

Interviewed by Ellen P. Neff

Published online: 20 April 2020 https://doi.org/10.1038/s41684-020-0549-6 